\title{
OPEN Macrophage morphological plasticity and migration is Rac signalling and MMP9 dependant
}

\author{
Jana Travnickova ${ }^{1,2}$, Sandra Nhim ${ }^{1}$, Naoill Abdellaoui ${ }^{1}$, Farida Djouad ${ }^{3}$, Maï Nguyen-Chi ${ }^{4}$, \\ Andrea Parmeggiani ${ }^{1,5}$ \& Karima Kissa ${ }^{1 凶}$
}

In vitro, depending on extracellular matrix (ECM) architecture, macrophages migrate either in amoeboid or mesenchymal mode; while the first is a general trait of leukocytes, the latter is associated with tissue remodelling via Matrix Metalloproteinases (MMPs). To assess whether these stereotyped migrations could be also observed in a physiological context, we used the zebrafish embryo and monitored macrophage morphology, behaviour and capacity to mobilise haematopoietic stem/progenitor cells (HSPCs), as a final functional readout. Morphometric analysis identified 4 different cell shapes. Live imaging revealed that macrophages successively adopt all four shapes as they migrate through ECM. Treatment with inhibitors of MMPs or Rac GTPase to abolish mesenchymal migration, suppresses both ECM degradation and HSPC mobilisation while differently affecting macrophage behaviour. This study depicts real time macrophage behaviour in a physiological context and reveals extreme reactivity of these cells constantly adapting and switching migratory shapes to achieve HSPCs proper mobilisation.

\author{
Abbreviations \\ AGM Aorta-gonad-mesonephros \\ CHT Caudal haematopoietic tissue \\ DA Dorsal aorta \\ HSPC Haematopoietic stem and progenitor cells \\ MMP Matrix metalloproteinases \\ PCV Posterior cardinal vein \\ TAM Tumour associated macrophages \\ HPF Hour Post Fertilization \\ ECM Extra Cellular Matrix
}

Macrophages were for the first time identified as phagocytic cells responsible for pathogen elimination ${ }^{1}$. Over the past century, they were associated with homeostasis, innate and adaptive immune responses, inflammation, tissue remodelling and cytokine production ${ }^{2,3}$. Macrophages are the most plastic haematopoietic cells present in all tissues; their diversity depends upon their location, their morphology, their membrane receptors or surface markers ${ }^{2}$. Depending on tissue composition they infiltrate and environmental constraints, macrophages adopt different migration modes ${ }^{4}$. In the course of a three-dimensional (3D) migration, macrophages can either adopt an amoeboid or a mesenchymal migratory mode. In case of an amoeboid migration, cells take on a round or polarised shape and migrate through the extracellular matrix (ECM). Such a migration is Rho/Rock GTPases dependent. During mesenchymal migration, macrophages degrade the ECM through proteinases secretions (e.g. Matrix Metalloproteinases or MMPs) and cells take on an elongated shape. This second migratory mode is Rac GTPase signalling dependent ${ }^{4,5}$.

In mouse and human, macrophage characterization was mainly performed in vitro using bone marrow derived macrophages. Recently, the zebrafish model was used to resolve specific issues during the developmental process or to address accurate pathologies. The transparency of zebrafish embryos enables the live imaging and

\footnotetext{
${ }^{1}$ Emergence of Haematopoietic Stem Cells and Cancer, LPHI, CNRS, INSERM, Univ Montpellier, Montpellier, France. ${ }^{2}$ Present Address: MRC Human Genetics Unit, and CRUK Edinburgh Centre, Institute of Genetics and Cancer, Western General Campus, University of Edinburgh, Edinburgh EH4 2XU, UK. ${ }^{3}$ IRMB, Univ Montpellier, INSERM, Montpellier, France, Montpellier, France. "Mise en Place de L'immunité et Inflammation, LPHI, CNRS, INSERM, Univ Montpellier, Montpellier, France. ${ }^{5}$ Laboratoire Charles Coulomb, CNRS, Univ Montpellier, Montpellier, France. ${ }^{\boxplus}$ email: karima.kissa-marin@umontpellier.fr
} 
real time tracking of cell populations. We and other groups have shown that two main waves of macrophages emerge from primitive and definitive haematopoiesis during the zebrafish development ${ }^{6-8}$. The initial wave takes place between 18 and $25 \mathrm{~h}$ post fertilization (hpf) in the yolk sac ${ }^{6}$. The second wave occurs between 30 and $55 \mathrm{hpf}$ in the aorta-gonad-mesonephros (AGM) $)^{7,8}$ and generates the haematopoietic stem/progenitor cells (HSPCs) (1, $^{9}$ which later will differentiate into all blood cells including macrophages. Additionally, a transient hematopoietic wave is initiated in the posterior blood island, giving rise to the multilineage progenitor cells and erythromyeloid progenitors, which develop into both erythroid and myeloid cells ${ }^{11}$.

Recently, we demonstrated in vivo that primitive macrophages are crucial in the establishment of a definitive haematopoiesis ${ }^{12}$. Macrophages that accumulated in the trunk region, in the AGM between the dorsal aorta (DA) and the posterior cardinal vein (PCV) degrade the ECM located in the vicinity of HSPCs via matrix metalloproteinase 9 (MMP-9) secretions, thereby enabling them to migrate, enter the blood stream and colonise haematopoietic organs.

In the present study, we provide an extensive analysis of macrophages in zebrafish embryos. Using morphological analysis we were able to distinguish for the first time different macrophage subtypes in vivo. By combining morphological analysis with live imaging we succeeded to visualise the dynamic behavioural patterns of individual macrophages during their migration through the ECM.

\section{Results}

Macrophage shape heterogeneity in the zebrafish embryo. During the establishment of the definitive haematopoiesis, macrophages accumulated in the AGM in the vicinity of the DA and the PCV, between 30 and 60 hpf degrade the ECM surrounding HSPCs via Mmp-9 secretions which result in the mobilization of HSPCs $^{12}$. Using this physiological and optically accessible model, we analysed the shape and behaviour of proteolytic macrophages in order to establish a potential correlation. We first described the position and shape of macrophages in the AGM using the $k d r l: e G F P / / m p e g 1: m C h e r r y-F$ double transgenic lines where the GFP protein highlighted vessels and mCherry-F macrophage membranes (Fig. 1a,b). Figure 1a provides a schematic view of the vessel and macrophage position as shown in Fig. 1b. Using a $3 \mathrm{D}$ view (Fig. 1b) we were able to determine the position of macrophages (white arrows) in the outer layer of the vein wall between the vein and the aorta floor with different morphologies. The particle analysis of macrophages from a maximum projected confocal acquisitions enabled us to distinguish and quantify the various macrophage shapes. Three main morphological criteria were identified: circularity, roundness and elongation factor (Fig. 1c,d and Suppl. Table S1). They revealed the existence of 4 main shapes whose images are shown in Fig. 1c-e. We named these 4 subgroups-round (1), amoeboid (2), star-like (3) and elongated shape (4). While the round and elongated shapes had already been described in vitro, the two remaining shapes might represent either subgroups present in vivo or intermediate stages between round and elongated shapes. The main difference between the amoeboid and star-like shapes lied in the presence of a main axis, i.e. polarity (identified by elongation factor) in the amoeboid shape. The quantification of each shape revealed that amoeboid, star-like and elongated shapes were equally present whereas the round shape remained sparse (Fig. 1f).

Dynamics of macrophage migration in vivo. The analysis of macrophage shapes revealed the existence of four morphological subgroups distributed in the zebrafish AGM. To assess the behaviour of each macrophage subgroup, we imaged $\mathrm{Tg}$ (Mpeg1:mCherry) embryos over the course of one hour (acquisition every minute; Video 1). A colour-coded process was used to visualize cells movement from one z-plane to another one via a colour modification. We selected time frames in colour depth projection that illustrated the dynamics of macrophages able to adopt different shapes within fifteen minutes (Fig. $2 \mathrm{a}-\mathrm{f}$ and Video 1, colour code scale). The outlines represent the shape of macrophages in the imaged area at the 9th minute and enable us to draw a direct comparison with following time points. The colour depth projection of confocal imaging enabled us to determine the depth of macrophage positions in vivo and to demonstrate their ability to migrate in $3 \mathrm{D}$ patterns (Video 1). In vivo tracking of all macrophages within a 60 min timeframe demonstrated that no specific directionality was maintained during their migration (Fig. $2 \mathrm{~g}, \mathrm{n}=23$ ) as opposed to macrophages attracted to a wound site as an example of typical oriented migration (Fig. $2 \mathrm{~h}, \mathrm{n}=27$ ). The speed of migration remained the same in both cases (Fig. 2i). Subsequently, we quantified the evolution of macrophage shapes over time. Every single macrophage in the AGM reveals an ability to change shape within a very short time span (measured every $5 \mathrm{~min}$ ) and to pass repeatedly through distinct shape subgroups over a $30 \mathrm{~min}$ course (Fig. $2 \mathrm{j}, \mathrm{n}=10$ ). The round shape appeared less frequently than others and live imaging showed that cells often adopted a round shape under two specific conditions: during cell division or once the macrophage entered the bloodstream.

In order to determine if any of macrophage shapes is correlated with a higher velocity, we evaluated cell shape modification for each time point of imaging acquisition (every minute) and measured the distance travelled by each macrophage (Supplementary Fig. S1). We then linked the shape and the distance migrated. Surprisingly, even though we could not detect any significant difference between the instantaneous velocity and the shape (Supplementary Fig. S1a), we detect a significant increase of velocity when a change of shape occurred (Supplementary Fig. S1b). We therefore suggest that the dynamic of shape change is contributing to the macrophage migration in physiological conditions. In conclusion, macrophage real time imaging completes the characterisation of mesenchymal migrating macrophages and shows for the first time that they can adopt successive morphologies for their migration in the 3D matrix. Moreover, we highlighted for the first time that the macrophage morphological modification is directly associated with speed motility.

Rac inhibition modifies macrophage behaviour and function. Using in vivo imaging we showed that macrophages exhibited morphological plasticity during their migration. This high plasticity depended on 
a

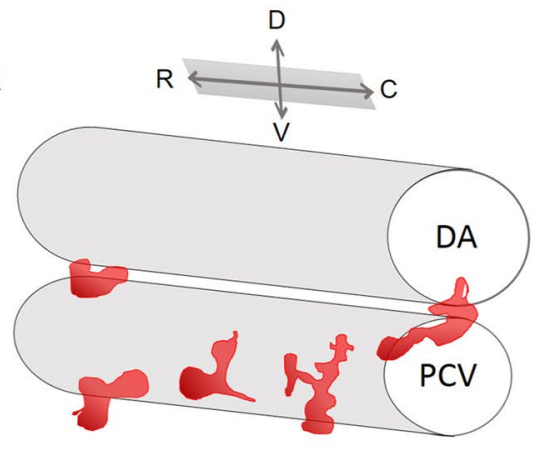

C

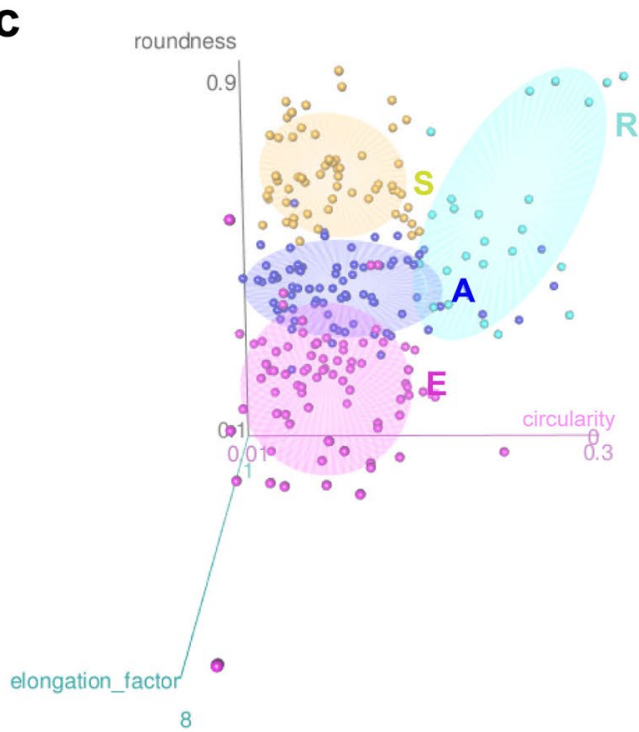

e

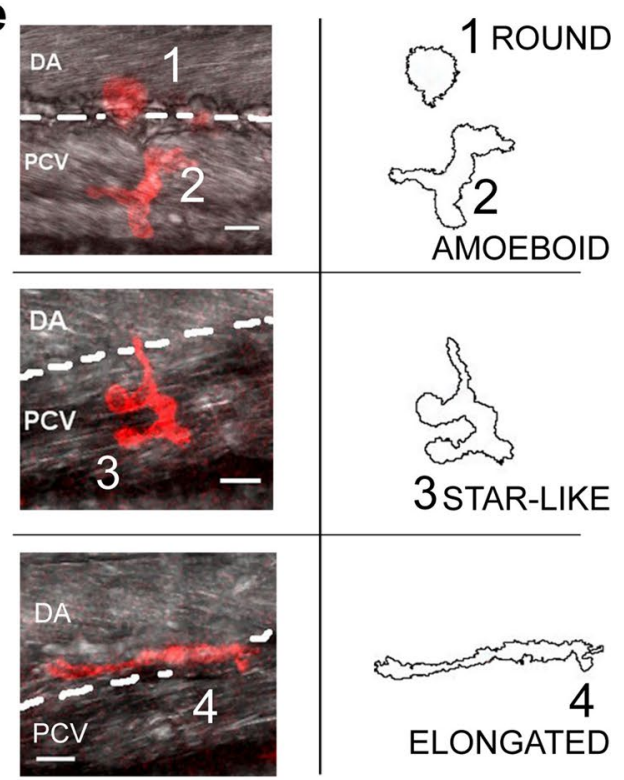

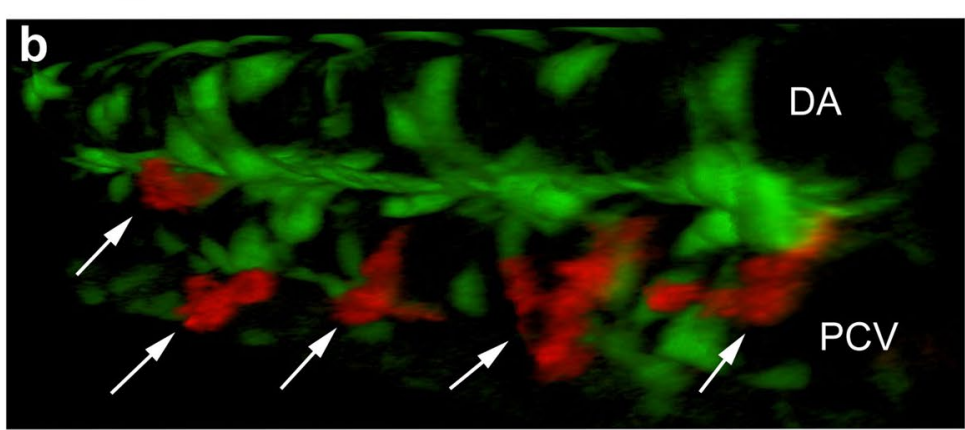

d

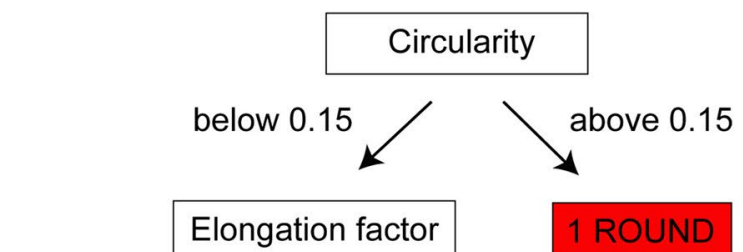

above 2.5

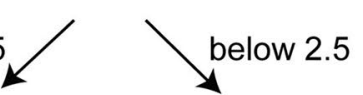

4 ELONGATED

\section{Roundness}

below 0.6

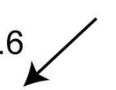

$\searrow^{\text {above } 0.6}$

2 AMOEBOID

3 STAR-LIKE

\section{AGM}

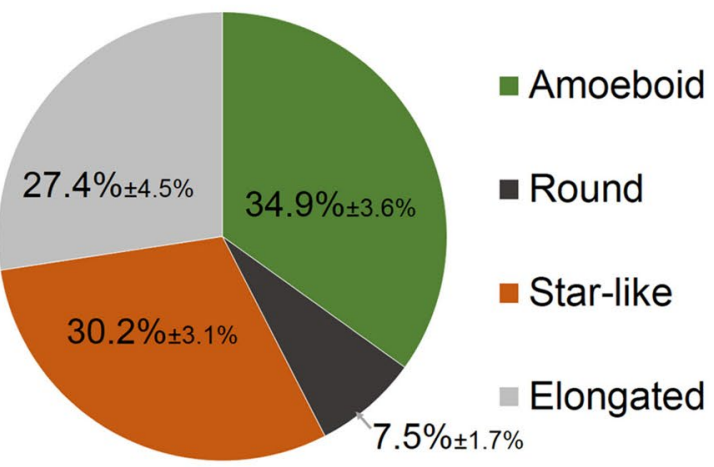

Figure 1. Macrophages in the AGM can be divided into 4 morphological subgroups. (a) The drawing shows a 3D view of vessels and macrophages (red) imaged in (b). (b) 3D view of the AGM in the mid-trunk region of a $T g(k d r l: e G F P / / m p e g 1: m C h e r r y-F)$ zebrafish embryo at $48 \mathrm{hpf}$ showing the position of vessels (endothelium in green) and macrophages (in red, white arrows) in the outer side of the vein and in between the dorsal aorta and the cardinal vein. (c) 3D scatter plot showing the 3 attributes (circularity, roundness and elongation factor) used to divide macrophage to their shape categories; plot was generated in R using rgl package ${ }^{25}$. (d) Diagram of the decision chart for the 4 categories of macrophages delineated according to shape attributes. (e) Representative confocal images (maximum intensity projections) of individual categories with an outline drawing from particle analysis on the right. (f) Graph representing the percentage distribution of the different shape categories per AGM. Data are represented as percentage average \pm s.e.m. $\mathrm{N}=20$ embryos. $C$ caudal, $D$ dorsal, $D A$ dorsal aorta, $P C V$ posterior cardinal vein, $R$ rostral, $V$ ventral. See also Supplementary table $S 1$. 

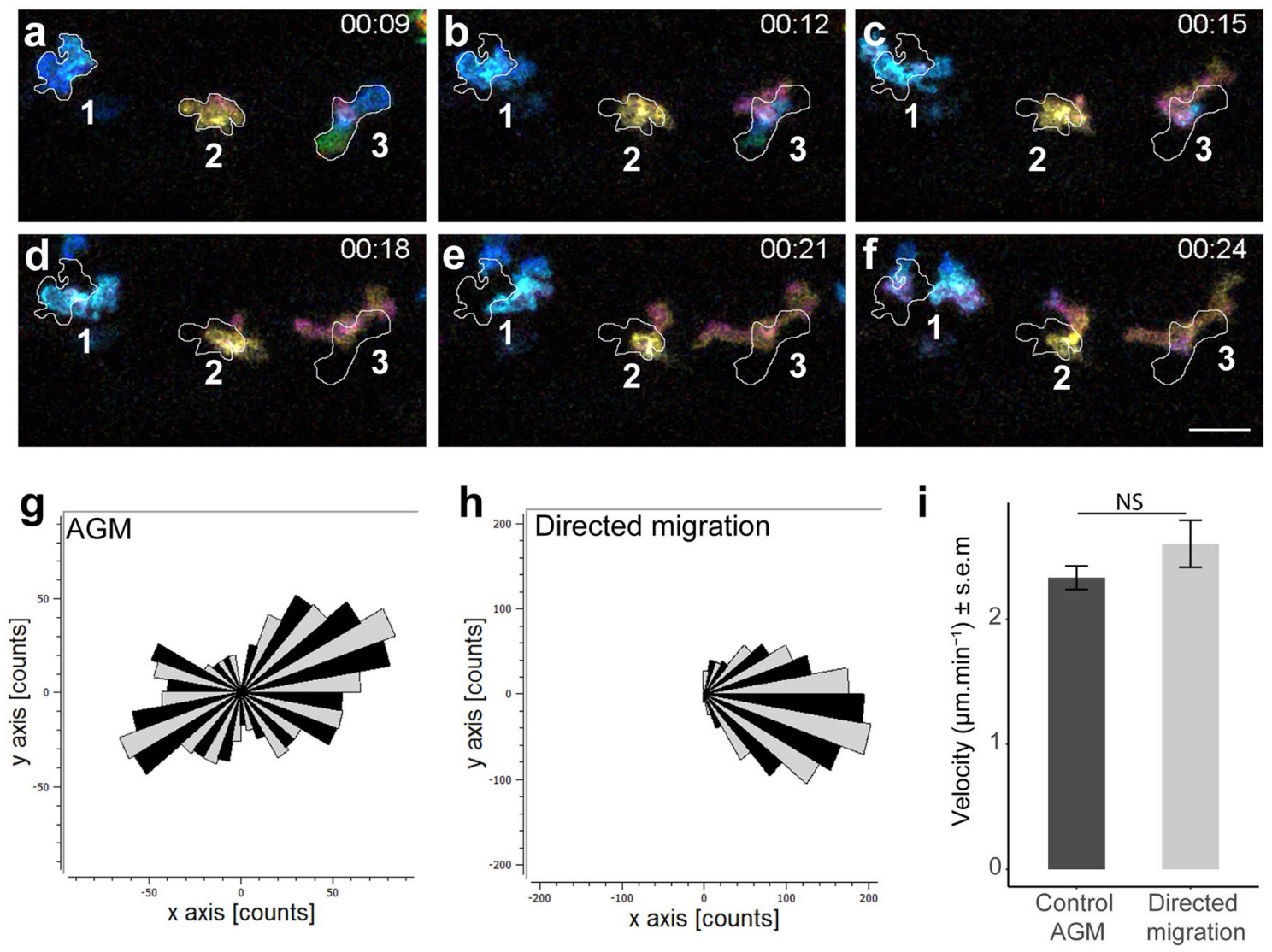

j

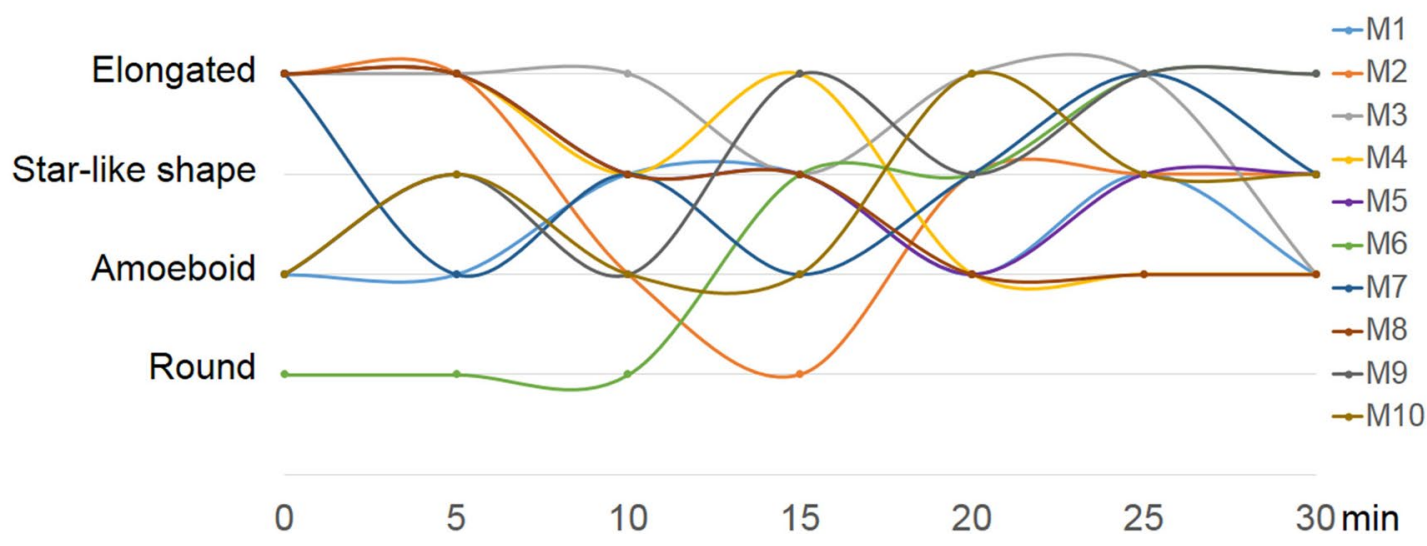

Figure 2. Macrophages in the AGM migrate in the mesenchymal way and undergo dynamic transition between different shapes over time. (a-f) Selected images from Video 1 illustrate the macrophage migration and shape transformation over time. Numbers point to individual macrophages. Time code is expressed in hours and minutes. White outlines on panel (b-f) indicate the shape and position of macrophages from panel (a) (9th minute). (g,h) Rose plot diagrams show the directionality of macrophage migration in the AGM compared to the oriented migration of macrophages in the tail region after tail fin cut injury. A diagram represents the single counts of the position of each macrophage in the selected area (black and grey sectors of angle $\pi / 18$ ) every minute over 60 min with a $(x, y, 0,0)$ starting point. $n=23$ macrophages for the control and $n=27$ for directed migration. (i) Bar plot show the comparable velocity between macrophages in the AGM and macrophages migrating toward a cut in the tail region. NS not significant; bar plot was generated in $\mathrm{R}$ using ggplot2 package. (j) Graph showing the shape evolution of individual macrophages during a 30 min course with 5 min interval measurements. Every line represents a single macrophage $(n=10)$. See also Video 1 . Scale bar $(\mathbf{a}-\mathbf{f}) 30 \mu \mathrm{m}$. See also Supplementary Figure S1. 
both, external (the stroma rigidity) and intrinsic parameters (cytoskeleton dynamics) ${ }^{4}$. One intrinsic factor associated with mesenchymal migration is the small GTPase- Rac signalling. We thus investigated the effect of Rac chemical inhibition on macrophage shape and migration patterns. The macrophage shape distribution in Rac inhibitor (NSC23766) treated embryos did not significantly differ from that of DMSO treated control (Fig. $3 \mathrm{a}, \mathrm{N}_{\mathrm{DMSO}}=10$ and $\mathrm{N}_{\text {Racinh. }}=14$ embryos). Selected images from Video 2 (colour-depth-projection, bottom, Fig. $3 \mathrm{~b}-\mathrm{e}$ ) demonstrated that the macrophage migration was much slower than that of control embryos (Video 2, top). Macrophage speed measured over $60 \mathrm{~min}$ in the AGM confirmed a decrease in velocity from $2.37 \pm 0.13$ to $1.13 \pm 0.16 \mu \mathrm{m} \mathrm{min}^{-1}$ (Fig. 3f).

The tracking plot diagram illustrated macrophage migration path and distance in control and Rac-inhibited embryos (Fig. 3g,h, $\mathrm{n}=15$ macrophages from 4 embryos, position measured every minute over $1 \mathrm{~h}$ ) and revealed that Rac inhibition reduced macrophage moves from $130.9 \pm 7.5$ to $56.8 \pm 7.8 \mu \mathrm{m}$. Moreover, the analysis of macrophage shape dynamics, revealed a reduction in macrophage plasticity over time as macrophages were no longer able to consecutively adopt different shapes (Fig. 3i, right; $n=7$ ) versus control (Fig. 3i, left; $n=7$ ). However, in spite of reduced plasticity levels, membrane extensions were still formed at the same rate and with similar length as in control macrophages. Rac inhibition resulted in an increase in single extension span (from $2.8 \pm 0.3$ to $11.0 \pm 1.9 \mathrm{~min}, \mathrm{n}_{\text {extension }}=45$, Supplementary Fig. S2a) as opposed to that of control macrophages.

As macrophage migration and morphological plasticity were significantly affected by Rac inhibition, we decided to evaluate the functionality of these macrophages. The main role of AGM macrophages is to degrade the ECM and to enable HSPC migration ${ }^{12}$. In vivo zymography of $T g(M p e g 1: m C h e r r y)$ embryos at 48 hpf, revealed a significant reduction in gelatin degradation and thereby a lower gelatinase activity (decreased number of green dots of cleavage-revealed FITC) in Rac inhibited embryos compared to control (Fig. 3j). Since the proteolytic function of macrophages in the AGM is essential to HSPC mobilisation, we assessed the effect of Rac inhibition on haematopoietic organ colonisation. We noticed an increase in HSPCs accumulated in the AGM at 48 hpf $(+70 \pm 8 \%, \mathrm{n}=6$, Supplementary Fig. S2b and consequently a decrease in HSPC accumulated in the caudal haematopoietic tissue (CHT) at $55 \mathrm{hpf}(-41 \pm 2 \% ; \mathrm{n}=5$, Supplementary Fig. S2c).

MMP inhibition affects macrophage shape, behaviour and function. Rac inhibition has an impact on macrophage proteolytic activity and consequently on their function. To assess whether direct inhibition of macrophage proteolytic activity induces a similar behaviour, we soaked embryos in a medium containing MMP inhibitor (SB-3CT, MMP-2 and -9 inhibitor). We previously demonstrated that ECM degradation occurred as a result of macrophage-secreted MMP-9 around HSPCs to enable their intravasation (12). We evaluated the direct impact of MMP inhibition on macrophage morphology and noticed a variation in shape distribution: an increase in round shape number and a decrease in star-like and elongated shapes (Fig. 4a). Moreover, MMP inhibition affected macrophage migration and behaviour (Fig. 4b-e, Video 3). Selected images from Video 3 displayed a typical example of macrophage migration pattern. Using Colour depth projection we were able to visualise the 3D migration of macrophages in the AGM and noticed that in MMP-9 inhibited embryos, macrophages migrated mainly in 2D. At a given point in time, they adopted a single colour whereas in control embryos we observed dynamic changes indicated by the presence of several colours at one time point (Fig. 2a-f). Moreover, Video 3 showed the macrophages adopted different migration pattern resembling to the leukocyte crawling on vein vessel.

Furthermore, we observed that MMP inhibition affected macrophage velocity and directionality. The speed of migration increased more than 3 times compared to the control (from $2.20 \pm 0.11$ to $7.80 \pm 0.92 \mu \mathrm{m} \mathrm{min}^{-1}$; Fig. 4f). Finally, a tracking plot diagram which illustrated the migration path and distance of macrophages in the AGM in control and MMP-inhibited embryos (Fig. 4g,h) revealed that migration directionality increased from 0.27 to 0.66 . Cell tracking showed that macrophages migrated along the vein, in the same direction as the blood flow. We concluded that, MMP inhibition affected both macrophage shape and migration patterns. They adopted a MMP independent migration pattern with increased velocity which was reminiscent of an amoeboid type of migration.

\section{Discussion}

In this study we characterised in zebrafish embryos the macrophage population present in the AGM with a known proteolytic function ${ }^{12}$. We reported the existence of four macrophage morphological subgroups. Previous studies performed in vitro described two major morphological types, elongated and rounded shapes ${ }^{13}$. Using in vivo analyses we were able to identify two additional morphological shapes: amoeboid and star-like shapes. In vivo observations revealed the presence of a higher number of macrophage subgroups in contrast to conclusions drawn from assays on $3 \mathrm{D}$ matrices, thereby suggesting the importance of in vivo modelling to complete results obtained in vitro. Using high resolution live imaging in conjunction with macrophage shape descriptor analysis we devised a novel tool that enabled to quantify in vivo the dynamics and morphological plasticity of macrophages.

While macrophages were thought to exclusively migrate using an amoeboid mode ${ }^{14}$, Dr Parini's group demonstrated their capacity to also use a mesenchymal migration mode ${ }^{15}$. In line with this last study, we describe the mesenchymal macrophage migration process in vivo in zebrafish embryos. Macrophages revealed an increase in shape plasticity which confirmed the outcome of previous studies performed in vitro ${ }^{15}$. Moreover, we demonstrated that the dynamic shape changes of macrophages are associated with the increased speed of migration.

Previous studies highlighted the significance of the role played by Rac signalling in cytoskeleton organisation during the mesenchymal migration of cells $s^{5}$. Our study performed in vivo during the establishment of haematopoiesis in zebrafish embryos also demonstrated that the mesenchymal migration of macrophages was Rac signalling dependent. Going further, we observed that Rac signalling inhibition affected not only macrophage 

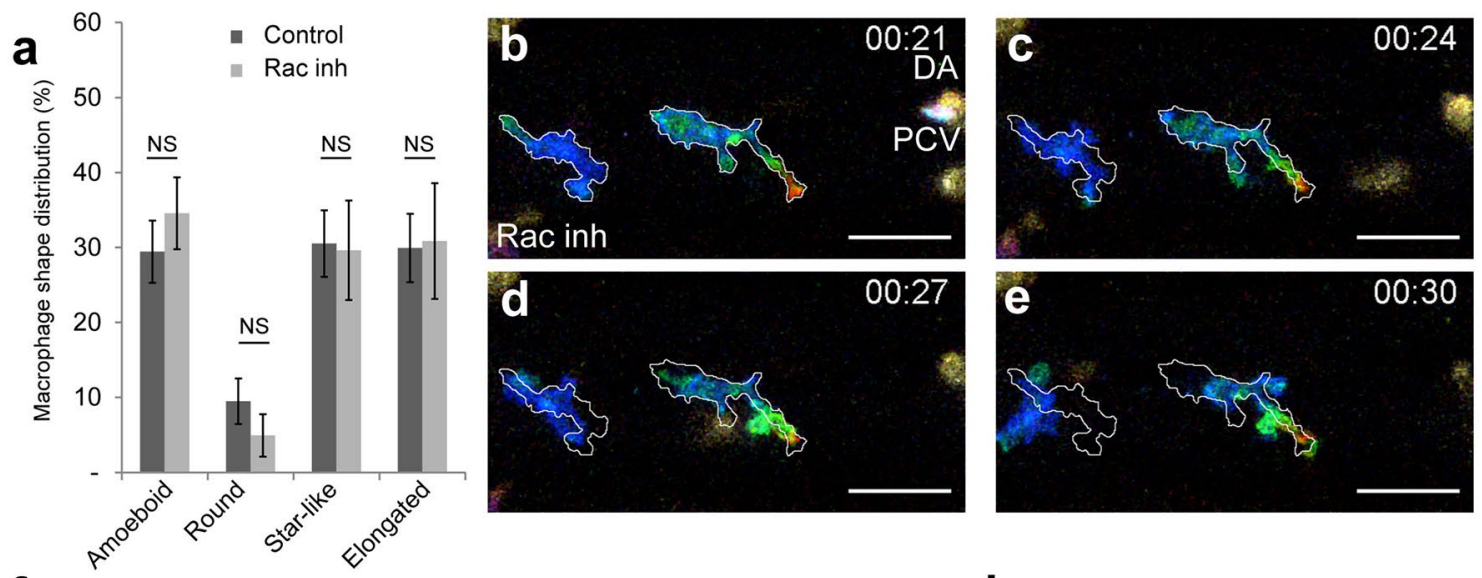

f

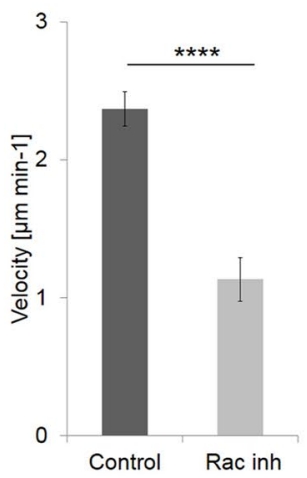

i

\section{g}

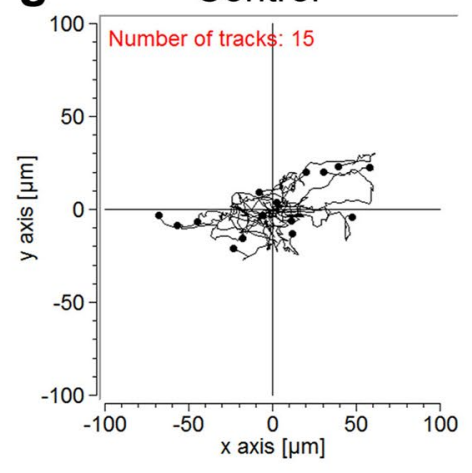

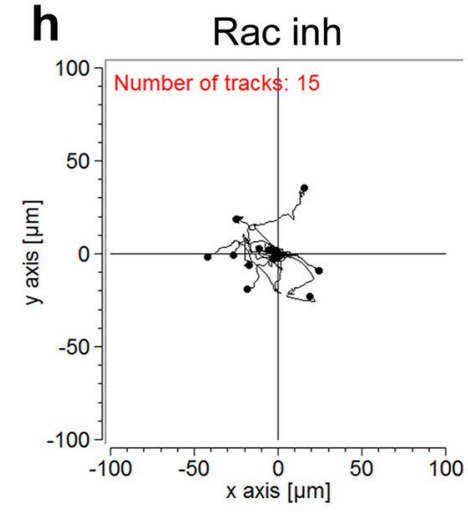

Rac inh

Control
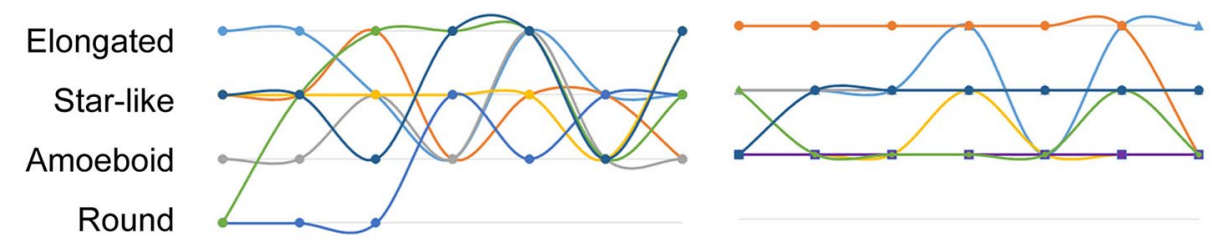

0

10

20

$30 \quad 0$

10

20

$30 \mathrm{~min}$

\section{$\operatorname{Tg}($ Mpeg1:mCherry) - Gelatin-FITC}
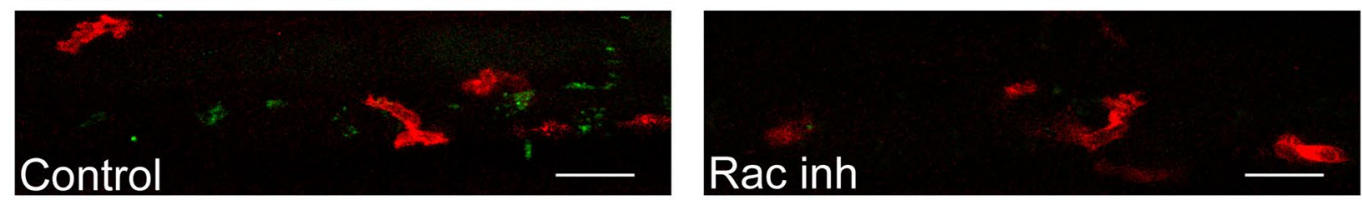

Figure 3. Rac inhibition leads to a loss of macrophage plasticity and motility. (a) Graph comparing macrophage shape distribution in the AGM of NSC23766 Rac-inhibited embryos (Rac Inh) to DMSO treated embryos (control) shows no significant change of distribution. $\mathrm{N}=10$ embryos for control and 15 for Rac inh. Data are represented as the mean of the percentage of each shape type in the total macrophage population in the AGM \pm s.e.m. NS = not significant. $(\mathbf{b}-\mathbf{e})$ Selected cropped images from Video 2 showing the shape and migration of macrophages over time of Rac inhibited embryo. Time code in hours and minutes. White outlines on panel $(\mathbf{c}-\mathbf{e})$ indicate the shape and position of macrophages from panel (b) (21st minute). (f) Graph showing the velocity of macrophages in control and Rac-inhibited embryos. Data are represented as a mean \pm s.e.m., $\mathrm{n}=15$ macrophages from 4 different embryos, ${ }^{* * * *} \mathrm{p}<0.0001$. (g,h) Tracking plot diagram representing the migration path and distance of macrophages in the AGM in control and Rac-inhibited embryos measured every minute for $60 \mathrm{~min}$. Scale in $\mu \mathrm{m}, \mathrm{n}=15$ macrophages from 4 different embryos. (i) Graph shows the shape evolution of individual macrophages during a $30 \mathrm{~min}$ course with $5 \mathrm{~min}$ interval measurements, Control to the left, Rac inhibitor to the right. Each line represents a single macrophage $(n=7)$. Statistically significant differences exist in the number of shapes adopted during a 30 min measurement course $(\mathrm{P}=0.003)$ as well as in the number of changes between two different shapes $(\mathrm{P}=0.006)$. (j) In vivo zymography in $\mathrm{Tg}(\mathrm{Mpeg1:mCherry)}$ embryos at $48 \mathrm{hpf}$ reveals the degradation of inserted gelatin (green dots of cleavage-revealed FITC) in control embryos and a highly reduced degradation after Rac inhibition. See also Video 2. Scale bar $30 \mu \mathrm{m}$. See also Supplementary Figure S2. 

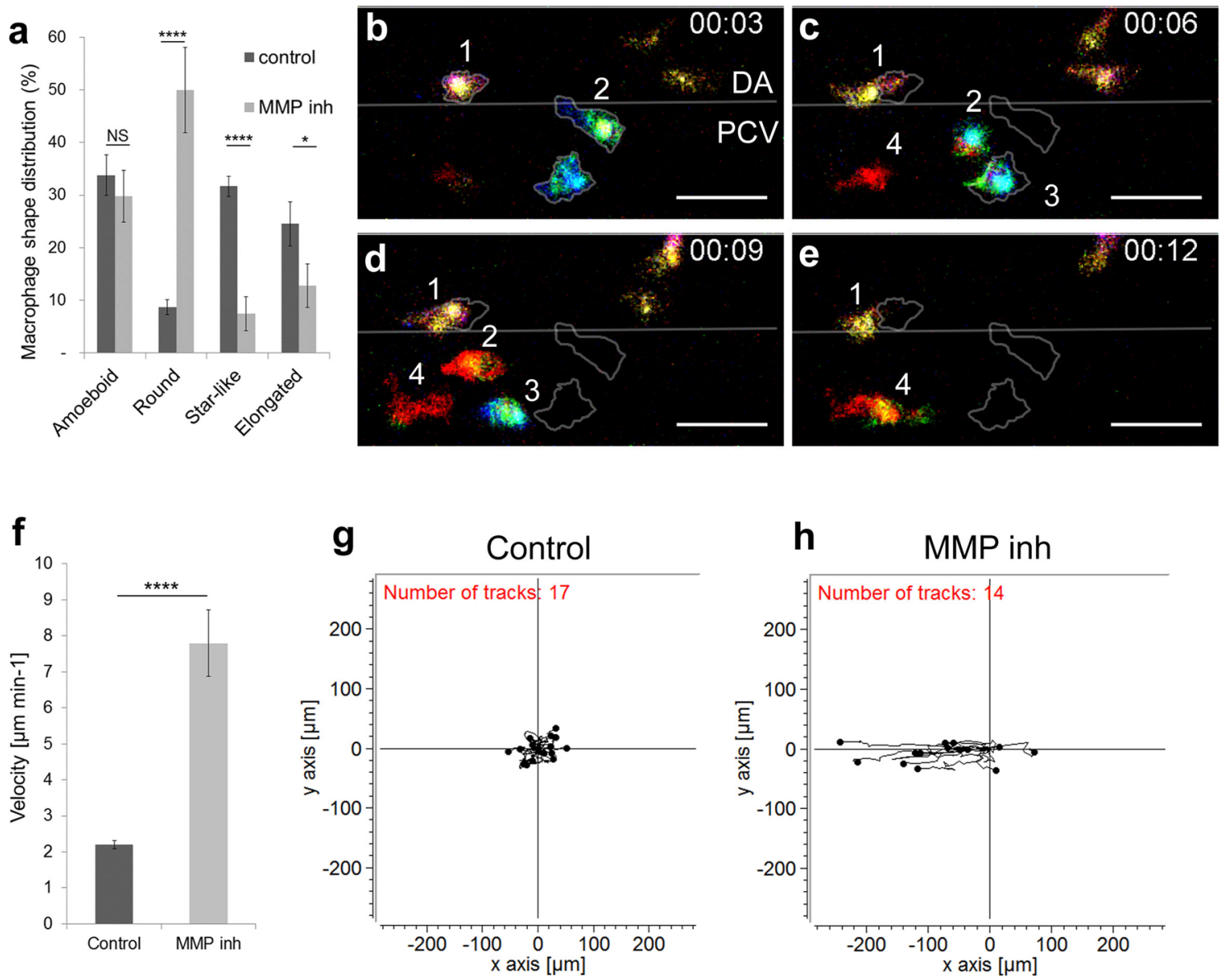

Figure 4. MMP-9 inhibition induces a change in macrophage shape and a transition towards an amoeboidlike migration. (a) Graph compares the macrophage shape distribution in the AGM of MMP-2 and 9 (SB-3CT) -inhibited embryos (MMP inh) to DMSO treated embryos (control) shows an increase in round shape and a decrease in star-like and elongated shapes in MMP inh embryos. N=10 embryos for control and 15 for MMP inh. Data represent the percentage mean for each shape type out of the total number of macrophages in the AGM \pm s.e.m. NS not significant; ${ }^{*} \mathrm{p}<0.05 ;{ }^{* * *} \mathrm{p}<0.0001$. (b-e) Selected cropped images from Video 3 displays macrophage shape and migration patterns over time. Numbers point to individual macrophages, time code is expressed in hours and minutes. Grey outlines on panel C-E show the shape and position of macrophages from panel (b) (3rd minute). (f) Graph showing the velocity of macrophages in control and MMP-inhibited embryos. Data are represented as a mean \pm s.e.m., $n=17$ macrophages for control and 14 for MMP inhibitor from 4 different embryos, ${ }^{* * *} \mathrm{p}<0.0001$. (g,h) Tracking plot diagram represents the migration path and distance of macrophages in the AGM in control and MMP-inhibited embryos measured every minute over $60 \mathrm{~min}$. Scale in $\mu \mathrm{m}, \mathrm{n}=17$ macrophages for control and 14 for MMP inhibitor from 4 different embryos. See also Video 3. Scale bar $30 \mu \mathrm{m}$. DA dorsal aorta, $P C V$ posterior cardinal vein.

migration but also their proteolytic function and their phenotype. Indeed, upon Rac inhibition macrophages lose their ability to degrade the ECM matrix. We also observed that this treatment significantly reduced macrophage velocity and morphological plasticity. This is in line with the data obtained when we analysed morphology changes and macrophage speed. We concluded that the speed motility is directly associated with macrophage morphological modification. Moreover, live imaging revealed that macrophages develop and keep longer membrane extensions and that they remained longer in a specific location. Our study confirmed previous in vitro observations showing that Rac1-/- macrophages cultured on plastic exhibited additional membrane extensions when compared to control macrophages ${ }^{16}$.

We finally observed that the inhibition of the macrophage proteolytic function induces their transition into a different type of migration mode corresponding to the adaptation of macrophages to their new environment. They adopted a round shape with an amoeboid migration. Macrophages were no longer able to migrate within the AGM stroma and they moved along the vein wall.

Proteolytic macrophages in the AGM exhibited a high functional similarity to macrophages found in solid tumours referred to as tumour associated macrophages (TAM). TAM play a significant part in ECM remodelling through proteinase releases (mainly MMP-2 and 9) and allow tumour cells to join the bloodstream and to seed in secondary $\operatorname{sites}^{17}$. Therefore, a current strategy is to target TAM to combat cancer ${ }^{18}$. Several approaches based on macrophage depletion (clodronate liposomes) or functional modification (broad spectrum MMP inhibitors) did 
not succeed and failed during clinical trials due to low specificity and the amount of side effects ${ }^{18,19}$. Expanding our knowledge from a purely molecular standpoint toward an in-depth understanding of behaviour and requirements in migration and site infiltration using adapted in vivo models, would complement existing studies and enable us to develop more targeted immunotherapeutic solutions.

\section{Materials and methods}

Zebrafish husbandry. Wild-type and transgenic lines were maintained in compliance with the Institutional Animal Care and Use protocols. The following transgenic lines were used in this study: Tg(Mpeg1:mCherry$F)^{12,20}$ for macrophage membrane marking and $\mathrm{Tg}(\mathrm{kdrl}: e G F P)^{21}$ for vessel endothelium labelling. Embryos were kept in the presence of 1-phenyl-2-thiourea to prevent melanin pigmentation ${ }^{22}$ and staged as described by Kimmel et al. ${ }^{23}$. All experiments were performed in accordance with the protocol CEEA-LR-13007 approved by the Animal Care and Use Languedoc-Roussillon Committee.

Live imaging. Zebrafish embryos (lateral views, rostral to the left) were embedded in $0.7 \%$ low melting agarose and imaged using a Zeiss LSM510 confocal microscope through a $40 \times$ water immersion objective with a $1024 \times 256$ pixel resolution at $28^{\circ} \mathrm{C}$. All live imaging experiments were performed at $46-48 \mathrm{hpf}$ and all time-lapse imaging occurred at an acquisition rate of one minute at a $1 \mu \mathrm{m} z$-interval. The acquisitions were performed using ZEN2009. Image processing such as maximum intensity projections, 3D view, and overall image contrast adjustment were performed using Fiji software.

Inhibitor treatment. Embryos were soaked in MMP-2 and 9 inhibitors SB-3CT (Enzo Life Sciences) $9 \mu \mathrm{M}$ or NSC23766 Rac inhibitor (Tocris) $50 \mu \mathrm{M}$ or DMSO $0.25 \%$ as a control from 5-prim stage (25 hpf) to $46-48$ hpf. For stock solution, inhibitors were dissolved in DMSO at a $10 \mathrm{mM}$ concentration and stored at $-20^{\circ} \mathrm{C}$.

Image processing and macrophage shape analysis. Confocal stacks of membrane-labelled macrophages were projected using a maximum intensity projection and $2 \mathrm{D}$ images were binarised using an automatic threshold. The following shape descriptors were evaluated using the Fiji plugin Particle analysis: area $\left(\mu \mathrm{m}^{2}\right)$, perimeter $(\mu \mathrm{m})$, circularity and roundness. The elongation factor was manually measured by dividing the longest axis of the object by its longest perpendicular axis $(\mathrm{x} / \mathrm{y})$. Objects with an area under $80 \mu \mathrm{m}^{2}$ were excluded from the further analysis. Circularity was calculated using the following formula: $4 \pi \times$ (area/perimeter $^{2}$ ). This parameter varied from 0 (linear polygon) to 1 (perfect circle). Circularity was used to set apart round objects (circularity $>0.15$ ) and roundness and elongation factor enabled us to break down non-round subjects into 3 subgroups: elongated, amoeboid and star-like shaped. Roundness was calculated using the following formula: $4 \times\left\{\right.$ area/ $\left.\left[\pi \times(\text { major axis) })^{2}\right]\right\}$ and varied from 0 (linear polygon) to 1 (perfect round). $3 \mathrm{~d}$ scatter plot of circularity, roundness and elongation actor in Fig. 1c was generated in R using rgl package ${ }^{24,25}$. Supplementary table S1 shows the mean values of circularity, roundness and elongation factor measured for each of the above listed subgroups.

Cell tracking and velocity measurement. Maximum intensity projections of $60 \mathrm{~min}$ time-lapses acquired every minute were analysed using a manual tracking plugin in Fiji. Measured data were transferred into a Chemotaxis and Migration tool programme (Ibidi) to design tracking and rose plots (Fig. 2g,h for rose plots, Figs. 3g,h and $4 \mathrm{~g}$,h for tracking plots). A rose diagram maps single counts of the position of every macrophage in a selected area (black and grey sectors of angle $\pi / 18$ ) every minute over 60 min with an $(x, y 0,0)$ starting point. The tracking plot diagram represents the migration path and distance of macrophages in the AGM with an $\mathrm{x}, \mathrm{y}$ 0,0 starting point, being measured every minute over $60 \mathrm{~min}$. The average of single macrophage velocities $(\mu \mathrm{m}$ $\mathrm{min}^{-1}$ ) during 15-60 min were used for analysis. The evaluation of the directionality was performed using a Rayleigh statistical test for the uniformity of a circular distribution of points (end points of single macrophages). All analyses were conducted using the Chemotaxis and Migration tool software (Ibidi).

Fin amputation for oriented migration analysis. Caudal fin amputation was performed with a sterile scalpel at $44 \mathrm{hpf}$, posterior to muscle and notochord under anaesthesia with $0.016 \%$ Tricaine (ethyl 3-aminobenzoate, Sigma Aldrich). $4 \mathrm{~h}$ post amputation embryos were mounted and imaged as described above.

In vivo zymography. The In vivo zymography was performed according to Crawford's protocol ${ }^{26}$. A working solution, $1 \mathrm{mg} \mathrm{ml}^{-1}$ of fluoresceinated gelatin (Gelatin-FITC,Anaspec) in PBS was injected (4-5 ng) into muscles between 4 and 5 th somite at $42 \mathrm{hpf}$. Imaging was performed following the injections. Embryos were incubated in DMSO or Rac inhibitor from $25 \mathrm{hpf}$ up to the Gelatin-FITC injections.

Statistical analysis. Normal distributions were analysed using the Shapiro-Wilk test. Non-Gaussian data were analysed using the Wilcoxon test, Gaussian with Student t-test. $\mathrm{P}<0.05$ was considered as statistically significant (symbols: ${ }^{* * *} \mathrm{p}<0.0001{ }^{* * *} \mathrm{p}<0.001{ }^{* *} \mathrm{p}<0.01{ }^{*} \mathrm{p}<0.05$ ) Statistical analyses were performed using the R software.

Ethics. All animal experiments described in the present study were conducted at the University of Montpellier in compliance with European Union guidelines for handling of laboratory animals (http://ec.europa. eu/environment/chemicals/lab_animals/home_en.htm) and the ARRIVE guidelines (https://arriveguidelines. 
org) and were approved by the Direction Sanitaire et Vétérinaire de l'Hérault and Comité d'Ethique pour l'Experimentation Animale under reference CEEA-LR-13007.

Received: 15 November 2020; Accepted: 5 April 2021

Published online: 12 May 2021

\section{References}

1. Metchnikoff, É. Leçons sur la pathologie comparée de l' inflammation : faites à l'Institut Pasteur en avril et mai 1891 / par Élie Metchnokoff. (Librairie de l'académie de médecine, 120, Boulevard Saint-Germain, Paris, 1892).

2. Wynn, T. A, Chawla, A. \& Pollard, J. W. Macrophage biology in development, homeostasis and disease. Nature 496, 445-55 (2013).

3. Gordon, S. \& Taylor, P. R. Monocyte and macrophage heterogeneity. Nat. Rev. Immunol. 5, 953-964 (2005).

4. Vérollet, C. et al. Extracellular proteolysis in macrophage migration: Losing grip for a breakthrough. Eur. J. Immunol. 41, 2805-2813 (2011).

5. Sanz-Moreno, V. \& Marshall, C. J. The plasticity of cytoskeletal dynamics underlying neoplastic cell migration. Curr. Opin. Cell Biol. 22, 690-696 (2010).

6. Herbomel, P., Thisse, B. \& Thisse, C. Ontogeny and behaviour of early macrophages in the zebrafish embryo. Development 126, 3735-3745 (1999).

7. Murayama, E. et al. Tracing hematopoietic precursor migration to successive hematopoietic organs during zebrafish development. Immunity 25, 963-975 (2006).

8. Gering, M. \& Patient, R. Hedgehog signaling is required for adult blood stem cell formation in Zebrafish embryos. Dev. Cell 8, 389-400 (2005).

9. Bertrand, J. Y. et al. Haematopoietic stem cells derive directly from aortic endothelium during development. Nature 464, 108-111 (2010).

10. Kissa, K. \& Herbomel, P. Blood stem cells emerge from aortic endothelium by a novel type of cell transition. Nature 464, 112-115 (2010).

11. Bertrand, J. Y. et al. Definitive hematopoiesis initiates through a committed erythromyeloid progenitor in the zebrafish embryo. Development 134, 4147-4156 (2007).

12. Travnickova, J. et al. Primitive macrophages control HSPC mobilization and definitive haematopoiesis. Nat. Commun. 6, 6227 (2015).

13. McWhorter, F. Y., Wang, T., Nguyen, P., Chung, T. \& Liu, W. F. Modulation of macrophage phenotype by cell shape. Proc. Natl. Acad. Sci. U. S. A. 110, 17253-17258 (2013).

14. Friedl, P. \& Weigelin, B. Interstitial leukocyte migration and immune function. Nat. Immunol. 9, 960-969 (2008).

15. Cougoule, C. et al. Blood leukocytes and macrophages of various phenotypes have distinct abilities to form podosomes and to migrate in 3D environments. Eur. J. Cell Biol. 91, 938-949 (2012).

16. Wheeler, A. P. et al. Rac1 and Rac2 regulate macrophage morphology but are not essential for migration. J. Cell Sci. 119, 2749-2757 (2006).

17. Condeelis, J. \& Pollard, J. W. Macrophages: obligate partners for tumor cell migration, invasion, and metastasis. Cell 124, 263-266 (2006).

18. Panni, R. Z., Linehan, D. C. \& Denardo, D. G. Targeting tumor-infiltrating macrophages to combat cancer. Immunotherapy 5, 1075-1087 (2013).

19. Turk, B. Targeting proteases: successes, failures and future prospects. Nat. Rev. Drug Discov. 5, 785-799 (2006).

20. Ellett, F., Pase, L., Hayman, J. W., Andrianopoulos, A. \& Lieschke, G. J. Mpeg1 promoter transgenes direct macrophage-lineage expression in Zebrafish. Blood 117, e49-56 (2011).

21. Beis, D. et al. Genetic and cellular analyses of zebrafish atrioventricular cushion and valve development. Development 132, 41934204 (2005).

22. Westerfield, M. The Zebrafish Book. A Guide for the Laboratory Use of Zebrafish (Danio rerio), 4th Edition. (2000).

23. Kimmel, C. B., Ballard, W. W., Kimmel, S. R., Ullmann, B. \& Schilling, T. F. Stages of embryonic development of the zebrafish. Dev. Dyn. 203, 253-310 (1995).

24. R Development Core team. R Core Team. R: A Language and Environment for Statistical Computing. R Foundation for Statistical Computing, Vienna, Austria. ISBN 3-900051-07-0. URL http://www.R-project.org/. vol. 55 275-286 (2015).

25. Adler, D. rgl: 3D Visualization Using OpenGL. R package version 0.105.22. (2021).

26. Crawford, B. D. \& Pilgrim, D. B. Ontogeny and regulation of matrix metalloproteinase activity in the zebrafish embryo by in vitro and in vivo zymography. Dev. Biol. 286, 405-414 (2005).

\section{Acknowledgements}

We would like to thank E. Lelièvre and M. Rossel for their critical review of this manuscript; V. Diakou and the MRI facility for their assistance, A. Sahuquet for his help in the particle analysis. This work was supported by grants from the ARC, Chercheur d'Avenir-Région Languedoc-Roussillon, FRM and ATIP-Avenir. J.T. was supported by a fellowship from the MESR and FRM (FDT20150532507), NA by a fellowship from the FRM, M.N.-C. by a fellowship from the Université de Montpellier.

\section{Author contributions}

J.T. and K.K. designed the project and the experiments, J.T., S.N. and N.A. performed the experiments and analysed the results. J.T. and K.K. wrote the manuscript with the input of S.N., M.N.-C., F.D. and A.P.

\section{Competing interests}

The authors declare no competing interests.

\section{Additional information}

Supplementary Information The online version contains supplementary material available at https://doi.org/ 10.1038/s41598-021-88961-7.

Correspondence and requests for materials should be addressed to K.K. 
Reprints and permissions information is available at www.nature.com/reprints.

Publisher's note Springer Nature remains neutral with regard to jurisdictional claims in published maps and institutional affiliations.

(c) (i) Open Access This article is licensed under a Creative Commons Attribution 4.0 International License, which permits use, sharing, adaptation, distribution and reproduction in any medium or format, as long as you give appropriate credit to the original author(s) and the source, provide a link to the Creative Commons licence, and indicate if changes were made. The images or other third party material in this article are included in the article's Creative Commons licence, unless indicated otherwise in a credit line to the material. If material is not included in the article's Creative Commons licence and your intended use is not permitted by statutory regulation or exceeds the permitted use, you will need to obtain permission directly from the copyright holder. To view a copy of this licence, visit http://creativecommons.org/licenses/by/4.0/.

(C) The Author(s) 2021 